\title{
Effect of a forbidden site on a $d$-dimensional lattice random walk
}

\author{
Nicolas Martzel * and Claude Aslangul ${ }^{\dagger}$ \\ Groupe de Physique des Solides, Laboratoire associé au CNRS UMR 7588, \\ Universités Paris 7 \& Paris 6, Tour 23, Place Jussieu, 75251 Paris Cedex 05, France
}

(October 29, 2018)

\begin{abstract}
We study the effect of a single excluded site on the diffusion of a particle undergoing random walk in a $d$-dimensional lattice. The determination of the characteristic function allows to find explicitly the asymptotical behaviour of physical quantities such as the particle average position (drift) $\langle\vec{x}\rangle(t)$ and the mean square deviation $\left\langle\vec{x}^{2}\right\rangle(t)-\langle\vec{x}\rangle^{2}(t)$. Contrarily to the one-dimensional case, where $\langle\vec{x}\rangle(t)$ diverges at infinite times $\left(\langle\vec{x}\rangle(t) \sim t^{1 / 2}\right)$ and where the diffusion constant $D$ is changed due to the impurity, the effects of the latter are shown to be much less important in higher dimensions: for $d \geq 2,\langle\vec{x}\rangle(t)$ is simply shifted by a constant and the diffusion constant remains unaltered although dynamical corrections (logarithmic for $d=2$ ) still occur. Finally, the continuum space version of the model is analyzed; it is shown that $d=1$, is the lower dimensionality above which all the effects of the forbidden site are irrelevant.
\end{abstract}

\section{INTRODUCTION}

Interactions between diffusing particles are expected to play an important role in many systems of physical interest (zeolites 1 , biological membranes 4 , one-dimensional hopping conductivity 4 ), especially in low dimension and even with short-range interactions. For $d=1$ the case of a contact interaction has been extensively studied in the past, following the pioneering paper by Harris on the so-called tracer problem, first showing that the mean-square displacement $\left\langle x^{2}\right\rangle$ increases as $t^{1 / 2}$ at large times. This result was also obtained by van Beijeren et al. 6 and, more recently, by Rödenbecket al. $\mathrm{Q}$; these authors indeed solve the full $N$-particle problem for an arbitrary initial condition and recover Harris' result by going to the $N \rightarrow+\infty$ limit. Still in the case of a zero-range repulsive interaction, asymptotic results (transport coefficients and distribution laws) in the extreme case of a finite- $N$ compact initial cluster have been obtained in refl, where the relation with the theory of extreme events was also discussed $\mathrm{B}$.

Obviously, the effects of short-range interactions are expected to become less and less important in higher dimensions (on the other hand, the interplay between interaction range and space dimensionality remains, as far as we know, an open question). In the present paper, we address the simplest problem, namely that of two particles with a contact interaction undergoing $d$-dimensional lattice random walk. After transforming to the center-of-mass frame, this essentially reduces to the Brownian motion of a single particle subjected to an isolated reflecting barrier. The equations for the general $d$-dimensional case are given below and, among other things, allow to show that $d=1$ is indeed the marginal dimensionality above which the random walk is unaffected by the presence of the localized impurity in the continuous version of the model; by this, it is meant that transport coefficients, as deduced from the dominant term in the asymptotic regime of physical quantities, are the same with and without impurity for $d>1$. Nevertheless, corrections still occur in the lattice model; that these corrections, less and less relevant as $d$ increases, basically display a $a^{d}$ behaviour for $d>1$, where $a$ denotes the lattice spacing.

This paper is organized as follows. We first write down the basic equations for any dimension $d$ and give the expression of the Laplace transform of the generating function giving by derivations all the moments of the particle coordinate in the lattice. We then analyze this general result, retrieving well-known results for the $d=1$ case. Subsequently, we examine in details the two-dimensional case, which exhibits lattice-dependent logarithmic corrections, and give the main results for the $d>2$ case. Even in such high dimensions, the presence of the forbidden site has consequences on the asymptotical dynamics: the final average particle position displays a shift $\delta x_{\mathrm{f}}$ which scales as $a^{d} x_{0}^{-d+1}$ where $x_{0}$ is the distance between the starting point of the particle and the exclusion site. Finally, the space-continuous version solution is written in full, showing that the moment-generating function is, in this limit, insensitive to the presence of the excluded site as far as the dimensionality $d$ is strictly greater that one.

\footnotetext{
*e-mail: martzel@gps.jussieu.fr

${ }^{\dagger}$ e-mail: aslangul@gps.jussieu.fr
} 


\section{BASIC EQUATIONS FOR THE $D$-DIMENSIONAL LATTICE WALK}

In the following, we consider a particle undergoing random walk in the viscous limit on a $d$-dimensional hypercubic lattice having a forbidden site. Denoting $\vec{e}_{p}$ the unit-vector in the $p$-th direction of the lattice, an arbitrary site of the lattice is fully specified by its vector $\vec{n}=\sum_{p=1}^{d} n_{p} \vec{e}_{p}$; the excluded site is located at the origin. Let $P(\vec{n}, t)$ be the probability to find the particule at site $\vec{n}$ at time $t$. In the continuous time description, it is readily seen that the time evolution of $P(\vec{n}, t)$ is governed by the following master equation:

$$
\frac{\mathrm{d}}{\mathrm{d} t} P(\vec{n}, t)=W[1-\delta(\vec{n}, \overrightarrow{0})] \sum_{\varepsilon= \pm 1} \sum_{p=1}^{d}\left[1-\delta\left(\vec{n}+\varepsilon \vec{e}_{p}, \overrightarrow{0}\right)\right]\left[P\left(\vec{n}+\varepsilon \vec{e}_{p}, t\right)-P(\vec{n}, t)\right]
$$

where $W$ denotes the probability per unit time to jump from one site to any of its nearest-neighbours; in (1),$\delta(\vec{n}, \vec{m})$ equals 1 if $\vec{n}=\vec{m}$ and vanishes otherwise; obviously, $\dot{P}(\vec{n}=0, t)=0$ at all times. The easiest way to solve this problem is to deal with the characteristic function of the probability distribution:

$$
\psi(\vec{\phi}, t)=\sum_{\vec{n}} \mathrm{e}^{\mathrm{i} \vec{\phi} \cdot \vec{n}} P(\vec{n}, t)
$$

which allows to find the probability function $P$ by the inverse formula :

$$
P(\vec{n}, t)=\int \mathrm{d}^{d} \phi \mathrm{e}^{-\mathrm{i} \vec{\phi} \cdot \vec{n}} \psi(\vec{\phi}, t)
$$

where $\phi_{p}=\vec{\phi} \cdot \vec{e}_{p}$ and where $\int \mathrm{d}^{d} \phi$ means $(2 \pi)^{-d} \int_{-\pi}^{+\pi} \mathrm{d} \phi_{1} \int_{-\pi}^{+\pi} \mathrm{d} \phi_{2} \ldots \int_{-\pi}^{+\pi} \mathrm{d} \phi_{d}$. Multiplying both sides of (值) by $\mathrm{e}^{\mathrm{i} \vec{\phi} \cdot \vec{n}}$ and summing over $\vec{n}$, one readily obtains:

$$
\frac{\partial}{\partial t} \psi(\vec{\phi}, t)=2 W \sum_{p=1}^{d}\left(\cos \phi_{p}-1\right) \psi(\vec{\phi}, t)+W \sum_{\varepsilon= \pm 1} \sum_{p=1}^{d}\left(\mathrm{e}^{\mathrm{i} \varepsilon \phi_{p}}-1\right) \int \mathrm{d}^{d} \phi^{\prime}\left(\mathrm{e}^{-\mathrm{i} \varepsilon \phi_{p}^{\prime}}-1\right) \psi\left(\vec{\phi}^{\prime}, t\right) .
$$

For sake of simplicity, the initial position of the walker is chosen to be $\vec{x}_{0}=n_{0} a \vec{e}_{1}$. With this condition, and since there is no external bias, the probability distribution is invariant under a mirror transformation through any plane perpendicular to $\vec{e}_{p}$ with $p \neq 1$. In addition, the quantities $\int \mathrm{d}^{d} \phi^{\prime} \cos \phi_{p}^{\prime} \psi\left(\overrightarrow{\phi^{\prime}}, t\right), p \neq 1$, are all equal. Introducing the Laplace transform of $\psi(\vec{\phi}, t)$ :

$$
\tilde{\psi}(\vec{\phi}, z)=\int_{0}^{+\infty} \mathrm{d} t \mathrm{e}^{-z t} \psi(\vec{\phi}, t)
$$

one readily obtains:

$$
\begin{aligned}
\left(z+2 W \sum_{p=1}^{d}\left(1-\cos \phi_{p}\right)\right) \tilde{\psi}(\vec{\phi}, z) & =\psi_{0}+2 W \sum_{p=2}^{d}\left(\cos \phi_{p}-1\right) \int \mathrm{d}^{d} \phi^{\prime}\left(\cos \phi_{p}^{\prime}-1\right) \tilde{\psi}\left(\overrightarrow{\phi^{\prime}}, z\right) \\
& +2 W \int \mathrm{d}^{d} \phi^{\prime}\left[\left(\cos \phi_{1}-1\right) \cos \phi_{1}^{\prime}+\sin \phi_{1} \sin \phi_{1}^{\prime}\right] \tilde{\psi}\left(\vec{\phi}^{\prime}, z\right)
\end{aligned}
$$

where $\psi_{0} \equiv \psi(\vec{\phi}, 0)=\mathrm{e}^{\mathrm{i} n_{0} \phi_{1}}$. Equation $(\sqrt{6})$ is a homogeneous separable Fredholm system, and can be solved by quadratures (see Appendix A), yielding the expression of $\tilde{\psi}(\vec{\phi}, z)$ (see eq. A8). For further reference, we explicitly write down the Laplace transforms of the average coordinate and mean-square displacement:

$$
\left\langle\tilde{x_{p}}\right\rangle(z)=-\left.\mathrm{i} a \frac{\partial \tilde{\psi}(\vec{\phi}, z)}{\partial \phi_{p}}\right|_{\vec{\phi}=\overrightarrow{0}}, \quad\left\langle\tilde{x_{p}^{2}}\right\rangle(z)=-\left.a^{2} \frac{\partial^{2} \tilde{\psi}(\vec{\phi}, z)}{\partial \phi_{p}^{2}}\right|_{\vec{\phi}=\overrightarrow{0}}
$$

With the solution given by $\mathrm{A} 8$, one precisely has:

$$
\left\langle\tilde{x_{p}}\right\rangle(z)=\delta_{p 1} \frac{a}{z}\left[n_{0}-\mathrm{i} \tilde{S_{1}}(Z)\right]
$$




$$
\left\langle\tilde{\vec{x}}^{2}\right\rangle(z)=\frac{a^{2}}{z}\left[\frac{d}{Z}+n_{0}^{2}+\tilde{C}_{1}(Z)+(d-1) \tilde{C}(Z)\right]
$$

where $Z=z /(2 W)$ and where the functions $\tilde{S}_{1}, \tilde{C_{1}}$ and $\tilde{C}$ are defined in Appendix A. In the next sections, we shall find the large-time behaviour of these quantities according to the dimensionality $d$, by analyzing their small $Z$ expansions $\left(Z \ll 1 \Longleftrightarrow t \gg W^{-1}\right)$.

\section{THE ONE-DIMENSIONAL CASE}

The reduced problem is here simply the $1 d$ diffusion of a particle submitted to a reflecting barrier. Although the result is well known, we shall discuss it to stress the main difference between this case and that of larger dimensionality. For $d=1$ the relevant $\tilde{C}_{1}$ function (A4) greatly simplifies $\left(\tilde{C}_{1}=\beta_{n_{0}} /\left(1-a_{1}-\beta_{1}\right)\right)$ whereas $\tilde{S}_{1}$ is formally unchanged and still given by (A4). Let first us discuss the drift term, given by (8). By using 10 :

$$
\int_{0}^{\infty} \mathrm{d} x \mathrm{e}^{-\alpha x} \mathrm{I}_{n}(x)=\frac{1}{\left(\alpha+\sqrt{\alpha^{2}-1}\right)^{n} \sqrt{\alpha^{2}-1}}, \quad \int_{0}^{\infty} \mathrm{d} x x^{-1} \mathrm{e}^{-\alpha x} \mathrm{I}_{n}(x)=\frac{\left(\alpha-\sqrt{\alpha^{2}-1}\right)^{n}}{n},
$$

we find that $\gamma_{n}$ (introduced in (A7)) is well-defined for all $z, \Re z \geq 0$ and indeed has a finite limiting value, namely $\gamma_{n}(z=0)=1$; this is one peculiarity of the one-dimensional case, for which $\gamma_{n} \simeq 1-\sqrt{2 Z}$ at small $Z$, so that $\tilde{S}_{1}$ diverges in the limit $Z \rightarrow 0$. In higher dimensions, the modulus of $\gamma_{n}$ is always stricty smaller than 1 for all $Z$, so that $\tilde{S}_{1}$ no more diverges in the limit $Z \rightarrow 0$. This property is crucial since it drastically changes the behaviour of the drift term according to the dimensionality, setting a marked difference between the cases $d=1$ and $d>1$ (see also section $\mathrm{Q}$, where the continuum limit is analyzed). This fact points out $d=1$ as being the marginal dimension for the drift term. A detailed calculation yields:

$$
\tilde{S}_{1}(z) \simeq \mathrm{i}\left\{\frac{1}{\sqrt{2 Z}}+\frac{1}{2}-n_{0}+\left[\frac{n_{0}}{2}\left(n_{0}-1\right)-\frac{1}{8}\right] \sqrt{2 Z}\right\} .
$$

Similarly:

$$
\tilde{C}_{1}(z) \simeq \frac{1}{\sqrt{2 Z}}+\frac{1}{2}-n_{0}+\left[\frac{n_{0}}{2}\left(n_{0}-1\right)-\frac{3}{8}\right] \sqrt{2 Z} .
$$

Using (11) and (12), the small- $z$ expansions of $\left\langle\tilde{x_{1}}\right\rangle(z)$ and $\left\langle\tilde{x_{1}^{2}}\right\rangle(z)$ can now be written out. Performing then the Laplace inversion yields the following asymptotic expansions:

$$
\begin{gathered}
\left\langle x_{1}\right\rangle(t) \sim 2 a \sqrt{\frac{W t}{\pi}}+\frac{a}{2}+\frac{\left(2 x_{0}-a\right)^{2}}{8 a \sqrt{\pi W t}}+\mathcal{O}\left(t^{-1}\right), \\
\left\langle x_{1}^{2}\right\rangle(t) \sim 2 a^{2} W t+2 a^{2} \sqrt{\frac{W t}{\pi}}+\left(x_{0}^{2}-a x_{0}+\frac{a^{2}}{2}\right)+\frac{1}{8}\left(4 x_{0}^{2}-4 a x_{0}-3 a^{2}\right) \frac{1}{\sqrt{\pi W t}}+\mathcal{O}\left(t^{-1}\right) .
\end{gathered}
$$

Note that the initial condition $x_{0}$ does not appear in the two first-dominant terms. The mean square deviation for the coordinate $x_{1}$ results:

$$
\Delta x_{1}^{2}(t) \equiv\left\langle x_{1}^{2}\right\rangle(t)-\left[\left\langle x_{1}\right\rangle(t)\right]^{2} \sim 2\left(1-\frac{2}{\pi}\right)\left[a^{2} W t+\frac{1}{2}\left(x_{0}-\frac{a}{2}\right)^{2}\right]+\mathcal{O}\left(t^{-1}\right) .
$$

The continuum limit is obtained from the lattice model by taking the limit $W \rightarrow \infty, a \rightarrow 0, n_{0} \rightarrow+\infty$ with $a^{2} W=D$ and $n_{0} a=x_{0}$ finite; performing this, one finds:

$$
\left\langle x_{1}\right\rangle(t) \sim 2 \sqrt{\frac{D t}{\pi}}+\frac{x_{0}^{2}}{2 \sqrt{\pi D t}}+\mathcal{O}\left(t^{-1}\right), \quad \Delta x_{1}^{2}(t) \sim 2\left(1-\frac{2}{\pi}\right)\left(D t+\frac{x_{0}^{2}}{2}\right)+\mathcal{O}\left(t^{-1}\right) .
$$

Note that the continuous limit strongly modifies the asymptotic expansions: in this limit, the first sub-dominant term of the lattice model drops out and the first relative corrections to the asymptotic leading term are $\mathcal{O}\left(t^{-1}\right)$ instead of 
$\mathcal{O}\left(t^{-1 / 2}\right)$ in the lattice version. On another point of view, comparison with (14) and 13) shows that the first lattice corrections vanish as the first power of the lattice constant $a$.

The above expressions, obtained as the continuous limits of the lattice model, can be compared to the wellknown results directly obtained in the continuous framework. For a particle subjected to a reflecting barrier and starting a distance $x_{0}>0$ apart, an elementary calculation yields the moment-generating function in the continuous version, which reads:

$$
\psi(k, t)=\frac{1}{2}\left[\psi_{+}(k, t)+\psi_{-}(k, t)\right] \quad \psi_{ \pm}(k, t)=\left[1+\Phi\left( \pm \frac{x_{0}}{\sqrt{4 D t}}+\mathrm{i} k \sqrt{D t}\right)\right] \mathrm{e}^{-D k^{2} t \pm \mathrm{i} k x_{0}},
$$

where $\Phi$ is the Error function 10 . By successive derivations at $k=0$, the two first moments result:

$$
\left\langle x_{1}\right\rangle(t)=x_{0} \Phi\left(\frac{x_{0}}{\sqrt{4 D t}}\right)+\sqrt{\frac{4 D t}{\pi}} \mathrm{e}^{-x_{0}^{2} /(4 D t)} \quad\left\langle x_{1}^{2}\right\rangle(t)=2 D t+x_{0}^{2} .
$$

These expressions are exact for all $t$; by an expansion at large times, they precisely reproduce (16), as it must.

\section{THE CASE $D \geq 2$}

The expected irrelevance of the forbidden site as dimension is increased will now be explicitly displayed. Among other things, it will be seen that, for $d=2,\left\langle\vec{x}^{2}\right\rangle$ and $\Delta \vec{x}^{2}$ contain logarithmic corrections to the "bare" diffusive regime, which become constant in time for $d>2$. These results allow to state that $d=2$ is indeed the marginal dimension for the diffusion constant characterizing the dominant term of the asymptotic behaviour. On the other hand, a permanent shift of the coordinate persists for any $d$, even at very large times.

Let us begin with the average coordinate $\left\langle x_{1}\right\rangle$. Contrarily to the case $d=1$, the integral $\gamma_{1}$ is always strictly smaller than one, since $\mathrm{e}^{-(Z+1) x} I_{n}(x)<1$ for all $Z$ and $x$ real. Using results given in the Appendix $\mathrm{B}$, one eventually obtains the following expression for the Laplace transform of the average coordinate valid for $|z| \ll W$ :

$$
\left\langle\tilde{x_{1}}\right\rangle(z) \simeq \frac{x_{0}}{z} \frac{4+\left(2 / n_{0}^{2}\right)-\left(n_{0}-1\right) Z \ln Z}{4-n_{0} Z \ln Z},
$$

which gives:

$$
\left\langle x_{1}\right\rangle(t) \sim\left(x_{0}+\frac{a^{2}}{2 x_{0}}\right)\left[1-\frac{1}{16 W t}\right]+\ldots \equiv x_{0}+\delta x(t)
$$

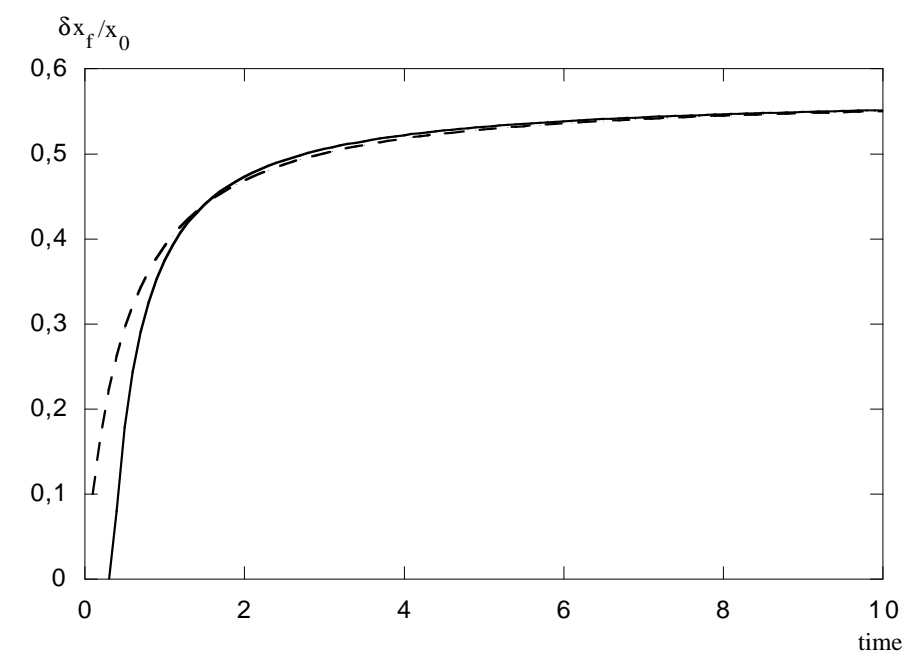

FIG. 1. Comparison for the final shift coordinate $\delta x_{\mathrm{f}}$ between analytical (solid curve) and numerical results (dashed line), for $n_{0}=1$ and $d=2$. The unit of time is $W^{-1}$. 
The final value of the average coordinate is thus simply shifted by $\delta x_{\mathrm{f}}=a^{2} /\left(2 x_{0}\right)$, all the more since the latter starts close to the forbidden site. As an illustration we show on figure 1 the analytical (solid curve) result for the final shift, compared with the numerical calculation (dashed curve), in the case $n_{0}=1$. The second moment can be obtained from (A4), (A5) and (9), Using the results of Appendix B:

$$
\begin{gathered}
\left\langle\tilde{\vec{x}}^{2}\right\rangle(z) \simeq \frac{1}{z}\left[\frac{4 a^{2} W}{z}+x_{0}^{2}+\frac{(Z+2) a_{n_{0}}(Z, 2)}{1-Z a_{1}(Z, 2)}\right] . \\
\left\langle\tilde{\vec{x}}^{2}\right\rangle(z) \simeq \frac{1}{z}\left[\frac{4 a^{2} W}{z}+x_{0}^{2}-\frac{a^{2}}{\pi} \ln \frac{z}{2 W}\right] .
\end{gathered}
$$

Laplace inversion gives the dominant behaviour at large times:

$$
\left\langle\vec{x}^{2}\right\rangle(t) \sim 4 a^{2} W t+x_{0}^{2}+\frac{a^{2}}{\pi} \ln 2 W t+\ldots .
$$

From (20) and (23), one finds the mean square deviation:

$$
\Delta \vec{x}^{2}(t) \sim 4 a^{2} W t+\frac{a^{2}}{\pi} \ln 2 W t .
$$

At this point, we see here that the diffusion constant, defined as usually:

$$
D=\lim _{t \rightarrow+\infty} \frac{1}{2 d t} \Delta \vec{x}^{2}(t)
$$

is now unchanged by the impurity but a logarithmic correction occurs in the sub-dominant term, which can be viewed as the reminder of the change of $D$ when $d=1$ : the diffusion constant is unchanged but the first correction diverges infinitely slowly at infinite times. This is clearly a marginal correction: at times large enough, the two-dimensional diffusive regime is the same with or without impurity.

In the continuous limit, the expressions (20) and (24) give:

$$
\left\langle x_{1}\right\rangle(t) \sim x_{0}+\mathcal{O}\left(\frac{a^{2}}{x_{0}}\right), \quad \Delta \vec{x}^{2}(t) \sim 4 D t+\mathcal{O}\left(a^{2} \ln \frac{2 a^{2}}{D t}\right)
$$

Let us now turn to the case $d>2$, for which all the integrals occurring in the subdominant terms are strictly convergent for all $Z, \Re Z \geq 0$, whereas the dominant terms are exactly the same as in the absence of a localized impurity (indeed, all the integrands behave at most as $x^{-d / 2}$ at infinity). More precisely, one finds:

$$
\left\langle\tilde{x_{1}}\right\rangle(z) \simeq \frac{x_{0}}{z}\left[1+\frac{\xi\left(n_{0}, d\right)}{1-\xi(1, d)}\right]
$$

and:

$$
\left\langle\tilde{\vec{x}}^{2}\right\rangle(z) \simeq \frac{1}{z}\left(\frac{2 d a^{2} W}{z}+A\right)
$$

where $A$ is a constant and where the function $\xi(n, d)$ is defined in B13. It results:

$$
\left\langle x_{1}\right\rangle(t) \sim x_{0}\left[1+\frac{\xi\left(n_{0}, d\right)}{1-\xi(1, d)}\right], \quad \Delta \vec{x}^{2}(t) \sim 2 d a^{2} W t+A^{\prime},
$$

where $A^{\prime}$ is another constant. The correction to unity in the brackets clearly represents the microscopic exclusion phenomemon, which again gives a final shift of the particle position, due to the presence of the impurity. As shown in the Appendix B, (B17), the function $\xi\left(n_{0}, d\right)$ is approximately given by:

$$
\xi(n, d) \simeq(\sqrt{\pi} n)^{-d} \Gamma(d / 2) \quad(n \gg 1),
$$

where $\Gamma$ denotes the Euler Gamma function. Equations $(27)$ and $(30)$ show that the final coordinate shift has an algebraic behaviour $\left(\delta x \sim a^{d} x_{0}{ }^{-d+1}\right)$ as a function of the distance between the starting point and the forbidden site. On the other hand, the subdominant term in $\Delta \vec{x}^{2}$ turns out to be now a constant in time, instead of diverging logarithmically as is the case for $d=2$. 


\section{THE SPACE CONTINUOUS LIMIT}

Here and there in the above, we gave the continuum limits of the first moments, in order to emphasize the corrections in the asymptotic regimes which are due to the lattice. In this section, we explicitly show that the $d>1$ random walk in continuum space is, as a whole, unaffected at all times by the local impurity. The central quantities $\tilde{S_{1}}$ and $\tilde{C_{p}}$ only depend on the reduced variable $Z=z a^{2} /(2 D)$, pointing out that the continuum limit embodies the limit $Z \rightarrow 0$. Let us first consider the behaviour of $\tilde{S}_{1}$; using the results of the Appendix B, we find at the lowest order in $Z$ :

$$
\tilde{S}_{1}(Z) \simeq \mathrm{i} \frac{\pi^{-d / 2} \Gamma(d / 2) n_{0}^{-(d-1)}}{\left[1-\pi^{-\frac{d}{2}} \Gamma(d / 2)\right]-F_{d}(Z)}
$$

where:

$$
F_{1}(Z) \simeq-\sqrt{2 Z}, \quad F_{2}(Z) \simeq \frac{1}{2 \pi} Z \log Z, \quad F_{d>2}(Z) \simeq \text { Cte } \times Z .
$$

Here we clearly see that for the drift term, the marginal dimension is $d=1$ : the term $\tilde{S}_{1}$ is divergent at low $Z$ only for $d=1$ and remains finite in this limit for $d>1$. Since the drift only depends on $\tilde{S}_{1}$, we can conclude that, for $d=1,\left\langle x_{1}\right\rangle(t)-x_{0}=2 \sqrt{D t / \pi}$, whereas for higher dimension, $\left\langle x_{1}\right\rangle-x_{0}$ vanish as $a^{d}$ in the continuum limit. yields :

Generally speaking, it is easy to obtain the continuous limit of the characteristic function (A8). A few algebra

$$
\begin{gathered}
\tilde{\psi}(k, z)=\frac{1}{z+D k^{2}}\left(\psi_{0}+\mathrm{i} k \sqrt{\frac{D}{z}}+\mathcal{O}(a)\right) \quad(d=1), \\
\tilde{\psi}(\vec{k}, z)=\frac{1}{z+D k^{2}}\left(\psi_{0}+\mathcal{O}\left(a^{d}\right)\right) \quad(d>1) .
\end{gathered}
$$

Thus, when the continuum limit is performed, all the additional terms arising from the excluded site strictly vanish except for $d=1$. As a whole, it turns out that $d=1$ is the marginal dimension of the problem in its space continuous version.

As a final remark, let us note that the present work indeed solves the problem of two particles with a hard-core mutual repulsion (exclusion process), each of them having the same diffusion constant $D$. By separating the free diffusion of the center-of-mass - which undergoes a normal diffusion with a constant $D / 2-$, one is left with a reduced particle with a constant $2 D$ and subjected to a static excluded point, which is the problem fully solved above.

\section{CONCLUSIONS}

We have studied the effect of a forbidden site on the random walk on a hypercubic lattice according to the dimensionality $d$ of the latter. For the mean square dispersion of the coordinate, the diffusion constant $D$ defining the asymptotical dynamics is altered only when $d=1$; for $d=2$, logarithmic corrections $\sim \ln t$ occur, which become constant in time when $d>2$, whereas $D$ is unchanged. On the contrary, the average coordinate is sensitive to the impurity in all cases. For $d=1$, it goes to infinity at large times $\left(\langle x\rangle(t) \sim t^{1 / 2}\right)$; for $d \geq 2$, its limiting value is always finite and displays a shift $\delta x_{\mathrm{f}} \propto a^{d} x_{0}^{-d+1}$ as compared to its initial value $x_{0}$.

As expected, these effects are found to be less important in the continuous- space version of the problem. By considering the generating function of the moments, it was shown that the impurity is totally irrelevant except for $d=1$ : as far as $d>1$, the continuous-space generating function is exactly the same as for the ordinary random walk (the above-mentionned corrections for $d \geq 2$ are thus lattice effects). This allows to state that $d=1$ is the marginal dimension for the continuous-space problem.

All these results were obtained by assuming a contact interaction between the walker and the impurity. Obviously enough, it can be anticipated that a long-range interaction strongly alters the present conclusions. Up to our knowledge, the interplay between the dimensionality and the interaction range is, presently, an open question that clearly deserves further study.

We are indebted to Julien Vidal for his most valuable remarks and comments on the manuscript. 


\section{APPENDIX A: SOLUTION OF THE FREDHOLM EQUATION}

Equation (6) is a homogeneous separable Fredholm system and can be solved by quadratures. We set:

$$
G(\vec{\phi}, z)=\frac{1}{z+2 W \sum_{p=1}^{d}\left(1-\cos \phi_{p}\right)},
$$

and introduce the auxiliary dimensionless quantities:

$$
\tilde{C}_{p}(z)=2 W \int \mathrm{d}^{d} \phi \cos \phi_{p} \tilde{\psi}(\vec{\phi}, z), \quad \tilde{S}_{1}(z)=2 W \int \mathrm{d}^{d} \phi \sin \phi_{1} \tilde{\psi}(\vec{\phi}, z)
$$

Due to the chosen initial condition, the $(d-1)$ functions $\tilde{C}_{p}, p=2,3, \ldots, d$, are all equal and are simply denoted by $\tilde{C}$ in the following. Using the standard procedure for solving such a separable Fredholm equation, $\tilde{C}_{p}$ and $\tilde{S}_{1}$ are seen to be given by an inhomogeneous system, which can be readily written and solved. Then, using the identity $u^{-1}=\int_{0}^{+\infty} \mathrm{d} x \mathrm{e}^{-u x}, \Re u>0$, it turns out to be that all these quantities can be expressed in terms of integrals involving the Bessel functions of the second kind $I_{n}$. More precisely:

$$
a_{n}(Z, d)=\int_{0}^{+\infty} \mathrm{d} x \mathrm{e}^{-(Z+d) x} \mathrm{I}_{n}(x) \mathrm{I}_{0}^{d-1}(x) \quad b_{n n^{\prime}}(Z, d)=\int_{0}^{+\infty} \mathrm{d} x \mathrm{e}^{-(Z+d) x} \mathrm{I}_{n}(x) \mathrm{I}_{n^{\prime}}(x) \mathrm{I}_{0}^{d-1}(x),
$$

A somewhat lengthy but straightforward calculation gives:

$$
\begin{gathered}
\left.\tilde{C}_{1}=\frac{1}{D}\left[1+a_{1}-\beta_{1}-(d-2)\left(\alpha_{11}-a_{1}\right)\right] \beta_{n_{0}}+(d-1)\left(\alpha_{11}-a_{1}\right) \alpha_{n_{0} 1}\right], \quad \tilde{S}_{1}=\frac{\mathrm{i} \gamma_{n_{0}}}{1-\gamma_{1}}, \\
\tilde{C}_{p \neq 1} \equiv \tilde{C}=\frac{1}{D}\left[\left(1+a_{1}-\beta_{1}\right) \alpha_{n_{0} 1}+\left(\alpha_{11}-a_{1}\right) \beta_{n_{0}}\right] .
\end{gathered}
$$

The various quantities appearing in these expressions are defined as follows:

$$
\begin{gathered}
\left.D=\left[1+a_{1}-\beta_{1}-(d-2)\left(\alpha_{11}-a_{1}\right)\right]\left(1+a_{1}-\beta_{1}\right)-(d-1)\left(\alpha_{11}-a_{1}\right)^{2}\right], \\
\beta_{n}=\frac{1}{2}\left[a_{n-1}+a_{n+1}\right], \quad \gamma_{n}=\frac{1}{2}\left[a_{n-1}-a_{n+1}\right], \quad \alpha_{n n^{\prime}}=b_{n n^{\prime}}(Z, d-1) .
\end{gathered}
$$

With these definitions, the solution of the central equation (6) writes:

$$
\tilde{\psi}(\vec{\phi}, z)=G(\vec{\phi}, z)\left[\psi_{0}+\tilde{C}(z) \sum_{p=2}^{d}\left(\cos \phi_{p}-1\right)+\tilde{C}_{1}(z)\left(\cos \phi_{1}-1\right)+\tilde{S}_{1}(z) \sin \phi_{1}\right]
$$

and is the starting point of the analysis undertaken in the present work.

\section{APPENDIX B: ASYMPTOTICAL BEHAVIOUR OF MANY-BESSEL FUNCTIONS INTEGRALS}

In this paper we repeatedly have to estimate integrals of the kind:

$$
I(Z, d, \vec{n}, m)=\int_{0}^{\infty} \mathrm{d} x \mathrm{e}^{-(Z+d) x} I_{n_{1}}(x) \ldots I_{n_{d}}(x) x^{m},
$$

in the limit $Z \ll 1$, where $I_{n}(x)$ denotes a modified Bessel function and $\vec{n}=\left\{n_{1}, \ldots, n_{d}\right\}$. When $Z=0$, no singularity arises from the lower bound provided that $n_{1}+\ldots+n_{d}+m>-1$ ( this is always the case in this paper). On the other side $(x \rightarrow \infty)$, the integral is finite for $m<(d / 2)-1$. Our goal is to obtain the asymptotical behaviour of $I(Z, d, \vec{n}, m)$ for large $n_{i}$ (but numerical checks show that some of the following analytical results still hold true even for $n^{2}=\Sigma n_{i}^{2}=1$ ).

In particular, when $I(Z, d, \vec{n}, m)$ is divergent, the knowledge of the nature of the divergence for $Z \sim 0$ is required. Let us first introduce the generalized integrals : 


$$
I_{g}(Z, d, \vec{n}, m, \omega)=\int_{0}^{\infty} \mathrm{d} x \mathrm{e}^{-(Z+d) x} \mathrm{e}^{-\omega / x} I_{n_{1}}(x) \ldots I_{n_{d}}(x) x^{m}
$$

which share the same diverging properties than $I$ when $Z \ll 1$ and allow to find the latter by one or other of the following procedures:

$$
\begin{gathered}
I(Z, d, \vec{n}, m)=\lim _{\omega \rightarrow 0} I_{g}(Z, d, \vec{n}, m, \omega) \\
I_{g}(Z, d, \vec{n}, m-1, \omega)=-\frac{\partial}{\partial \omega} I_{g}(Z, d, \vec{n}, m, \omega) \\
I_{g}(Z, d, \vec{n}, m+1, \omega)=-\frac{\partial}{\partial Z} I_{g}(Z, d, \vec{n}, m, \omega) .
\end{gathered}
$$

Let us first examine the case $d$ even $(d=2 p)$. The first non-logarithmically diverging integral is $I_{g}(Z, 2 p, \vec{n}, p, \omega)$. The divergence near $Z=0$ arises from large values of $x$. In order to get for purpose a convenient asymptotical form of Bessel functions, we first use an integral representation of the latter, and approximate it by using a stationary-phase argument when both $n$ and $x$ are much greater than unity :

$$
I_{n}(x)=\frac{1}{2 \pi} \int_{-\pi}^{+\pi} \mathrm{d} \theta \mathrm{e}^{x \cos \theta+\mathrm{i} n \theta} \simeq \frac{1}{2 \pi} \int_{-\infty}^{\infty} \mathrm{d} \theta \mathrm{e}^{x\left(1-\frac{1}{2} \theta^{2}\right)+\mathrm{i} n \theta}=\frac{1}{\sqrt{2 \pi x}} \mathrm{e}^{x-\frac{n^{2}}{2 x}} \quad(x, n \gg 1) .
$$

This approximation being done, all the integrals $I_{g}$ and $I$ only depend on the length $\rho f$ the vector $\vec{n}$, simply denoted by $n$ in the following. Using the approximate expression $(\sqrt{\mathrm{B} 6})$, we obtain with help of 10 :

$$
I_{g}(Z, 2 p, \vec{n}, p, \omega) \simeq(2 \pi)^{-p} \int_{0}^{\infty} \mathrm{d} x \mathrm{e}^{-Z x} \mathrm{e}^{-\frac{\omega+n^{2} / 2}{x}}=(2 \pi)^{-p} \sqrt{\frac{4\left(\omega+n^{2} / 2\right)}{Z}} K_{1}\left(\sqrt{4\left(\omega+n^{2} / 2\right) Z}\right)
$$

$K_{1}$ denoting the Bessel function of the second kind.

For $d$ odd $(d=2 p+1)$ we choose $I_{g}(Z, 2 p+1, \vec{n}, p, \omega)$ as a generator; by the same way as above we find:

$$
I_{g}(Z, 2 p+1, \vec{n}, p, \omega) \simeq(2 \pi)^{-\left(p+\frac{1}{2}\right)} \int_{0}^{\infty} \mathrm{d} x \mathrm{e}^{-Z x^{2}} \mathrm{e}^{-\frac{\omega+n^{2} / 2}{x^{2}}}=(2 \pi)^{-\left(p-\frac{1}{2}\right)} \sqrt{\frac{\pi}{Z}} \mathrm{e}^{\left.-2 \sqrt{Z\left(\omega+n^{2} / 2\right.}\right)} .
$$

Expressions (B7) and (B8) are correct in the limit $Z \ll 1$ and $n^{2} \gg 1$. Using then (B4) and (B5), we are now able to calculate the asymptotical behaviour of the whole family of $I$ integrals.

As an example, let us consider $I(Z, 2,\{0, n\},-1)$, which occurs in the calculation of the average coordinate for $d=2$. Explicitly, one has:

$$
I(Z, 2,\{0, n\},-1)=\int_{0}^{\infty} \mathrm{d} x x^{-1} \mathrm{e}^{-(Z+2) x} I_{0}(x) I_{n}(x),
$$

and the method sketched above yields:

$$
I(Z, 2,\{0, n\},-1) \simeq \frac{1}{\pi n^{2}}+\frac{1}{2 \pi} Z \ln Z \quad(Z \ll 1, n \gg 1) .
$$

We numerically checked the value of the $Z$-independent constant; it turns out that for $n \geq 5,1 /\left(\pi n^{2}\right)$ gives the correct value with a relative error smaller than $\sim 10^{-3}$. On the other hand, we found no simple way to numerically check the validity of the first-order term in the expansion $(\overline{B 10})$, but one can analytically prove that it is correct for $I(Z, 2,\{0,1\},-1)$. Indeed, the integral $I(Z, 2,\{0,1\}, 1)$ is computed in 10 :

$$
I(Z, 2,\{0,1\}, 1)=\frac{1}{2 \pi}\left[\frac{Z^{\prime}}{Z^{\prime 2}-1} \mathbf{E}\left(\frac{1}{Z^{\prime}}\right)-\frac{1}{Z^{\prime}} \mathbf{K}\left(\frac{1}{Z^{\prime}}\right)\right]
$$

where $\mathbf{K}$ and $\mathbf{E}$ are the complete elliptic functions of the first and the second kind respectively, and where $Z^{\prime}=1+Z / 2$. After a few algebra, we find :

$$
\lim _{Z \rightarrow 0} Z I(Z, 2,\{0,1\}, 1)=\frac{1}{2 \pi}
$$


Setting:

$$
\xi(\vec{n}, d)=I(0, d, \vec{n},-1)
$$

(B12) entails:

$$
\lim _{Z \rightarrow 0} I(Z, 2,\{0,1\},-1)=\xi(\{0,1\}, 2)+\frac{1}{2 \pi} Z \ln Z+C_{1} Z+\ldots,
$$

which for $n=1$ is in agreement with (B10).

As an application, let us find the asymptotical behaviour of the function $\xi(n, d)$ defined by (B13), which is strongly related to the coordinate shift in any dimension. For $d=2 p$, we find (remember that $n=|\vec{n}|$ ):

$$
\xi(n, 2 p)=\left.(-1)^{p+1} \frac{\partial^{p+1}}{\partial \omega^{p+1}} I_{g}(Z, \vec{n}, 2 p, p, \omega)\right|_{Z=\omega=0} \simeq \pi^{-p}(p-1) ! n^{-2 p},
$$

whereas for $d=2 p+1$, one has:

$$
\xi(n, 2 p+1)=\left.(-1)^{p+1} \frac{\partial^{p+1}}{\partial \omega^{p+1}} I_{g}(Z, \vec{n}, 2 p+1, p, \omega)\right|_{Z=\omega=0} \simeq \pi^{-p} \frac{1.3 .5 \ldots(2 p-1)}{2^{p}} n^{-(2 p+1)} .
$$

These two definitions turn in fact into only one :

$$
\xi(n, d) \simeq \pi^{-\frac{d}{2}} \Gamma(d / 2) n^{-d},
$$

$\Gamma$ still denoting the Euler Gamma function. It is worth noting that this asymptotical behaviour, a priori only valid for $|\vec{n}| \gg 1$, is very rapidly convergent and actually holds true even for rather small values of $|\vec{n}|$, as shown by a few numerical checks.

${ }^{1}$ V. Kukla, J. Kornatowski, K. Demuth, I. Girnus, H. Pfeifer, L. V. C. Rees, S. Schunk, K. K. Unger and J. K. Kärger, NMR Studies of Single-File Diffusion in Unidimensional Channel Zeolites, Science, 272, 702 (1996).

${ }^{2}$ E. Nener, Science, Ions Channels for Communication Between and Within Cells, 256, 498 (1992).

${ }^{3}$ B. Sackman, Elementary Steps in Synaptic Transmission Revealed by Currents Through Single Ion Channels, Science, 256, 503 (1992).

${ }^{4}$ P. M. Richards, Theory of one-dimensional hopping conductivity and diffusion, Phys. Rev. B, 16, 1393 (1977).

${ }^{5}$ T. E. Harris, Diffusion with "collisions" between particles, J. Appl. Prob., 2, 323 (1965)

${ }^{6}$ H. van Beijeren, K. Kehr, and K. W. Kutner, Diffusion in Concentration Lattice Gas. III: Tracer Diffusion on a OneDimensional Lattice, Phys. Rev. A,28, 5711 (1983)

${ }^{7}$ C. Rödenbeck, J. Kärger and K. Hahn, Calculating exact propagators in single-file systems via the reflection principle, Phys. Rev. E, 57, 4382 (1998).

${ }^{8} \mathrm{Cl}$. Aslangul, Classical diffusion of $N$ interacting particles: General results and asymptotic laws, Europhys. Lett., 44, 284 (1998).

${ }^{9}$ E. J. Gumbel, Statistics of Extremes, (Columbia University Press, 1958).

${ }^{10}$ I. S. Gradshteyn and I. M. Ryzhik, Table of integrals, Series and Products (Academic Press, 1980). 